\title{
Optical Signal Processing in Silicon-Based Integrated Devices
}

\author{
C.Lacava $^{1, *}$, T.D. Bucio ${ }^{1}$, A.Z. Khokhar ${ }^{1}$, D.J. Richardson ${ }^{1}$, F. Parmigiani ${ }^{1}$, F. Gardes ${ }^{1}$, \\ M. Sorel ${ }^{2}$ and P. Petropoulos ${ }^{1}$ \\ ${ }^{1}$ Optoelectronics Research Centre, University of Southamtpon, SO17 1BJ, United Kingdom \\ ${ }^{2}$ School of Engineering, University of Glasgow, G12 8LP, Glasgow, United Kingdom \\ ${ }^{*}$ C.Lacava@soton.ac.uk
}

\begin{abstract}
We discuss the latest progress on the development of all-optical signal processing devices in Si-based platform. We discuss the realization of intra and inter-modal wavelengthconverters by using our internally developed si-rich-silicon nitride platform.
\end{abstract}

OCIS codes: (190.0190) Nonlinear optics, (130.7405) Wavelength conversion devices

\section{Introduction}

In order to cope with the unstoppable increase of network traffic volume and internet services, modern telecommunications require a novel class of sophisticated all-optical signal processing devices, that can process optical signals at $\mathrm{Tb} / \mathrm{s}$ speed, without the need of any electrical component. Silicon photonic components have now become sufficiently mature and devices such as silicon phase and intensity modulators, passive and wavelength division multiplexing (WDM) components are now routinely employed in optical settings [1,2]. Nonlinear silicon photonic components have been extensively studied in the past years. Silicon on insulator (SOI) waveguides show large third-order Kerr nonlinearities, even at moderate pump-power levels $[3,4]$. In principle, this allows for the realization of power efficient, all optical signal processing devices, and many functionalities have been already demonstrated [3,5]. However, the efficiency of such components is significantly limited by both linear and nonlinear losses present in silicon [3]. For this reason, other platforms have been considered, such as silicon nitride [6] or silicon-germanium [7]. Recently, we proposed and studied the adoption of silicon-rich silicon nitride as nonlinear material [7-9]. We showed that, by properly changing the material deposition conditions, the linear and nonlinear response of the waveguides can be tailored according to the targeted application, allowing for efficient nonlinear interaction without excessive linear and nonlinear losses.

In this work we discuss the latest progress that have been made on the development of all-optical intra- and intermodal wavelength converters based on silicon-rich silicon nitride platform, suitable for both intensity and phaseencoded signals.

\section{Silicon-rich silicon nitride wavelength converter for phase encoded signals}

In $[8,9]$ we reported on the development of the silicon-rich silicon nitride platform, used to realize optical devices discussed in this paper. The proposed platform aimed to increase the Kerr response of the material with respect to the stoichiometric silicon nitride, without introducing excess linear and nonlinear losses. In Fig. 1 we show the nonlinear
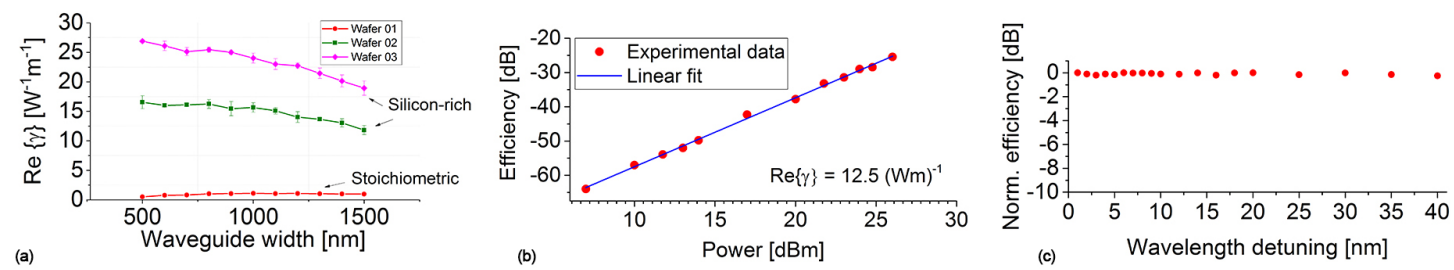

Fig. 1. (a)Nonlinear parameter Re $\gamma$ shown for different material composition, (b) FWM efficiency as a function of the pump power, (c) FWM bandwidth 

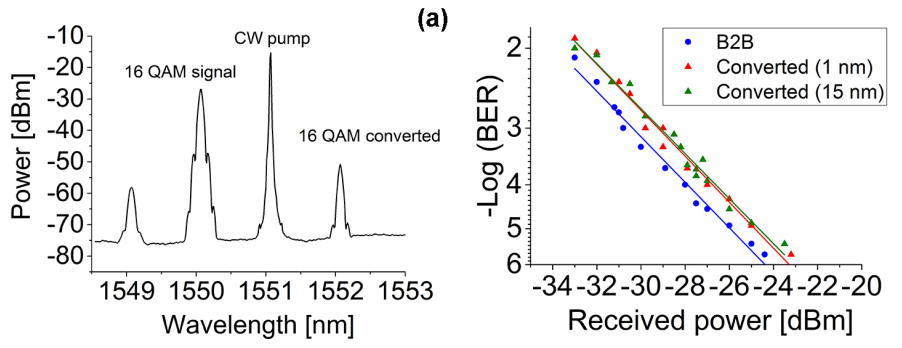

(b)

(c)

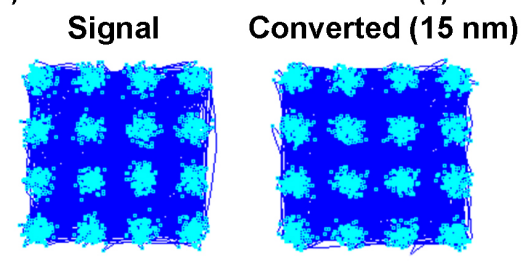

Fig. 2. (a)Example of FWM spectra, (B)BER measurements for the converted and the B2B signals (c) 16 QAM - constellation diagrams of the original and converted signals

parameter $\gamma$ measured on three different material compositions, including the standard, stoichiometric silicon nitride. As discussed in [9], the nonlinear parameter could be significantly enhanced (see Fig. 1(a), green curve). By further increasing the silicon content, the $R e \gamma$ reached values of $>26 \mathrm{Wm}^{-1}$, however this also led to unwanted losses. For this reason, the best trade-off was found for an intermediate material composition (green curve in Fig. 1(a), showing a linear refractive index of $n=2.54$ measured at $\lambda=1550 \mathrm{~nm}$ [9]. We realized the silicon-rich silicon nitride wavelength converter using a $1-\mathrm{cm}$ long, dispersion engineered waveguide, width a cross section of $1 \mu \mathrm{m} \times 0.7 \mu \mathrm{m}$, showing a zero dispersion wavelength at $\lambda=1549 \mathrm{~nm}$. The $\mathrm{CW}$-conversion efficiency behaviour of the waveguide under test is shown in Fig. 1(b) and 1 (c), as a function of the pump power and the pump-to-signal detuning, respectively. We tested the device in a real telecom setting, showing successful conversion of a 10 Gbaud-16 QAM signal. Results are shown in Fig. 2. BER penalties $<2 \mathrm{~dB}$ are obtained, even when the signal was detuned more than $15 \mathrm{~nm}$ from the pump, showing that this device can be used for broadband applications.

Results achieved using multiple spatial modes will be also presented at the conference. This novel scheme will allow obtaining broad- and wide-band wavelength conversion simultaneously, exploiting the various phase matching possibilities available when multi-mode structures are considered [10].

\section{Conclusion}

In this work we discussed the latest progress on the development of compact, telecom-compatible, wavelength converters, based on silicon-rich silicon nitride. At the conference, both intra- and inter-modal based converters will be discussed.

\section{References}

1. B. Jalali and F. Sasan, "Silicon photonics," Journal of Lightwave Technology 24, 4600-4615 (2004).

2. G. T. Reed, G. Mashanovich, F. Y. Gardes, and D. J. Thomson, "Silicon optical modulators," Nature Photonics 4 (2010).

3. J. Leuthold, C. Koos, and W. Freude, "Nonlinear silicon photonics," Nature Photonics 4, 535-544 (2010).

4. C. Lacava, M. Ettabib, I. Cristiani, J. Fedeli, D. Richardson, and P. Petropoulos, "Ultra-Compact Amorphous Silicon Waveguide for Wavelength Conversion," IEEE Photonics Technology Letters 28, 410-414 (2016).

5. C. Lacava, M. J. Strain, P. Minzioni, I. Cristiani, and M. Sorel, "Integrated nonlinear Mach Zehnder for 40 Gbit/s all-optical switching," Optics Express 21, 21,587 (2013).

6. Y. Okawachi, K. Saha, J. S. Levy, Y. H. Wen, M. Lipson, and A. L. Gaeta, “Octave-spanning frequency comb generation in a silicon nitride chip." Optics letters 36, 3398-3400 (2011).

7. C. Lacava, M. A. Ettabib, and P. Petropoulos, "Nonlinear Silicon Photonic Signal Processing Devices for Future Optical Networks," Applied Sciences 7, 103 (2017).

8. T. Domínguez Bucio, A. Z. Khokhar, C. Lacava, S. Stankovic, G. Z. Mashanovich, P. Petropoulos, and F. Y. Gardes, "Material and optical properties of low-temperature NH3-free PECVD SiNx layers for photonic applications," Journal of Physics D: Applied Physics 50 (2017).

9. C. Lacava, S. Stankovic, A. Z. Khokhar, T. D. Bucio, F. Gardes, G. T. Reed, D. J. Richardson, and P. Petropoulos, "Si-rich Silicon Nitride for Nonlinear Signal Processing Applications," Scientific Reports 7 (2017).

10. F. Parmigiani, P. Horak, Y. Jung, L. Grüner-Nielsen, T. Geisler, P. Petropoulos, and D. J. Richardson, “Alloptical mode and wavelength converter based on parametric processes in a three-mode fiber," Optics Express 25, 33,602 (2017). 